\title{
VALUE REGIONS OF UNIVALENT SELF-MAPS WITH TWO BOUNDARY FIXED POINTS
}

\author{
Pavel Gumenyuk ${ }^{\dagger}$ and Dmitri Prokhorov $\ddagger$ \\ University of Stavanger, Department of Mathematics and Natural Sciences \\ N-4036 Stavanger, Norway; pavel.gumenyuk@uis.no \\ Petrozavodsk State University, Lenina 33, 185910 Petrozavodsk, Russia \\ and Saratov State University, Department of Mathematics and Mechanics \\ Astrakhanskaya 83, 410012 Saratov, Russia; ProkhorovDV@info.sgu.ru
}

\begin{abstract}
In this paper we find the exact value region $\mathcal{V}\left(z_{0}, T\right)$ of the point evaluation functional $f \mapsto f\left(z_{0}\right)$ over the class of all holomorphic injective self-maps $f: \mathbf{D} \rightarrow \mathbf{D}$ of the unit disk $\mathbf{D}$ having a boundary regular fixed point at $\sigma=-1$ with $f^{\prime}(-1)=e^{T}$ and the Denjoy-Wolff point at $\tau=1$.
\end{abstract}

\section{Introduction}

Since the seminal paper [11] by Cowen and Pommerenke, the study of holomorphic functions with finite angular derivative at prescribed boundary points has been an active field of research in complex analysis, see, e.g., [2, 3, 10, 15, 17, 33, 38], just to mention some works in the topic.

Given a holomorphic function $f$ in the unit disk $\mathbf{D}:=\{z:|z|<1\}$ and a point $\sigma \in$ $\partial \mathbf{D}$ such that there exists finite angular limit $f(\sigma):=\angle \lim _{z \rightarrow \sigma} f(z)$, the angular derivative at $\sigma$ is $f^{\prime}(\sigma):=\angle \lim _{z \rightarrow \sigma}(f(z)-f(\sigma)) /(z-\sigma)$.

On the one hand, for univalent (i.e., holomorphic and injective) functions $f$, existence of the angular derivative $f^{\prime}(\sigma)$ different from 0 and $\infty$ is closely related to the geometry of $f(\mathbf{D})$ near $f(\sigma)$; moreover, if there exists $f^{\prime}(\sigma) \neq 0, \infty$, then the behaviour of $f$ at the boundary point $\sigma$ resembles conformality, see, e.g., [32, $\S \S 4.3,11.4]$.

On the other hand, for the dynamics of a holomorphic (but not necessarily univalent) self-map $f: \mathbf{D} \rightarrow \mathbf{D}$, a crucial role is played by the points $\sigma \in \partial \mathbf{D}$ for which $f(\sigma)=\sigma$ (or, more generally, $f(\sigma) \in \partial \mathbf{D})$ and the angular derivative $f^{\prime}(\sigma)$ is finite, see, e.g., $[5-7,8,9,14,16,31]$. Such points $\sigma$ are called boundary regular fixed points, see Section 2 for precise definitions and some basic theory. In particular, a classical result due to Wolff and Denjoy asserts that if $f \in \operatorname{Hol}(\mathbf{D}, \mathbf{D})$ has no fixed points in $\mathbf{D}$, then it possesses the so-called (boundary) Denjoy-Wolff point, i.e., a unique boundary regular fixed point $\tau$ such that $f^{\prime}(\tau) \leqslant 1$.

In this paper we study univalent self-maps $f: \mathbf{D} \rightarrow \mathbf{D}$ with a given boundary regular fixed point $\sigma \in \partial \mathbf{D}$ and the Denjoy-Wolff point $\tau \in \partial \mathbf{D} \backslash\{\sigma\}$. Using

https://doi.org/10.5186/aasfm.2018.4321 $30 \mathrm{C} 80$.

2010 Mathematics Subject Classification: Primary 30C75, 30D05; Secondary 30C35, 30C55,

Key words: Univalent function, value region, extremal problem, Denjoy-Wolff point, angular derivative, holomorphic self-map. 63699-P

${ }^{\dagger}$ Partially supported by Ministerio de Economía y Competitividad (Spain) project MTM2015-

${ }^{\ddagger}$ Supported by the Russian Science Foundation, project 17-11-01229, half a contribution. 
automorphisms of $\mathbf{D}$, we may suppose that $\tau=1$ and $\sigma=-1$. Our main result is the sharp value region of $f \mapsto f\left(z_{0}\right)$ for all such self-maps of $\mathbf{D}$ with $f^{\prime}(-1)$ fixed. To give a detailed statement, fix $z_{0} \in \mathbf{D}, T>0$ and let $\zeta_{0}=x_{1}^{0}+i x_{2}^{0}:=\ell\left(z_{0}\right)$, where

$$
\ell: \mathbf{D} \rightarrow \mathbf{S} ; \quad z \mapsto \log ((1+z) /(1-z)),
$$

is a conformal map of $\mathbf{D}$ onto the strip $\mathbf{S}:=\{\zeta:-\pi / 2<\operatorname{Im} \zeta<\pi / 2\}$. Define

$$
\begin{aligned}
a_{ \pm}(T) & :=e^{-T / 2} \sin x_{2}^{0} \pm\left(1-e^{-T / 2}\right), \quad R(a, T):=\log \frac{1-a}{1-a_{+}(T)} \log \frac{1+a}{1+a_{-}(T)}, \\
V\left(\zeta_{0}, T\right) & :=\left\{x_{1}+i x_{2} \in \mathbf{S}: a_{-}(T) \leqslant \sin x_{2} \leqslant a_{+}(T),\left|x_{1}-x_{1}^{0}-\frac{T}{2}\right| \leqslant \sqrt{R\left(\sin x_{2}, T\right)}\right\} .
\end{aligned}
$$

Theorem 1. Let $f \in \operatorname{Hol}(\mathbf{D}, \mathbf{D}) \backslash\left\{\mathrm{id}_{\mathbf{D}}\right\}$ and $T>0$. Suppose that

(i) $f$ is univalent in $\mathbf{D}$;

(ii) the Denjoy-Wolff point of $f$ is $\tau=1$;

(iii) $\sigma=-1$ is a boundary regular fixed point of $f$ and $f^{\prime}(-1)=e^{T}$.

Then

$$
f\left(z_{0}\right) \in \mathcal{V}\left(z_{0}, T\right):=\ell^{-1}\left(V\left(\ell\left(z_{0}\right), T\right)\right) \backslash\left\{z_{0}\right\} \quad \text { for any } z_{0} \in \mathbf{D} .
$$

This result is sharp, i.e., for any $w_{0} \in \mathcal{V}\left(z_{0}, T\right)$ there exists $f \in \operatorname{Hol}(\mathbf{D}, \mathbf{D}) \backslash\left\{\mathrm{id}_{\mathbf{D}}\right\}$ satisfying (i)-(iii) and such that $f\left(z_{0}\right)=w_{0}$.

We can also characterize functions $f$ delivering boundary points of $\mathcal{V}\left(z_{0}, T\right)$. In many extremal problems for univalent functions $f: \mathbf{D} \rightarrow \mathbf{C}$ normalized by $f(0)=$ $f^{\prime}(1)-1=0$, the Koebe function $f_{0}(z):=z /(1-z)^{2}$ mapping $\mathbf{D}$ onto $\mathbf{C} \backslash\left(-\infty, \frac{1}{4}\right]$, and its rotations $f_{\theta}(z)=e^{i \theta} f_{0}\left(e^{-i \theta} z\right), \theta \in \mathbf{R}$, are known to be extremal. For bounded univalent functions $f: \mathbf{D} \rightarrow \mathbf{D}$ normalized by $f(0)=0, f^{\prime}(0)>0$, the role of the Koebe function is played by the Pick functions $p_{\alpha}(z):=f_{0}^{-1}\left(\alpha f_{0}(z)\right), \alpha \in(0,1)$, mapping $\mathbf{D}$ onto $\mathbf{D} \backslash[-1,-r], r=r(\alpha) \in(0,1)$. In our case, it would be natural to expect that some functions of the form $f=h_{1} \circ p_{\alpha} \circ h_{2}$, where $h_{1}, h_{2} \in \operatorname{Aut}(\mathbf{D})$, are extremal.

Theorem 2. For any $w_{0} \in \partial \mathcal{V}\left(z_{0}, T\right) \backslash\left\{z_{0}\right\}$, there exists a unique $f=f_{w_{0}}$ satisfying conditions (i)-(iii) in Theorem 1 and such that $f_{w_{0}}\left(z_{0}\right)=w_{0}$. If $w_{0}=$ $\ell^{-1}\left(\zeta_{0}+T\right)$, then $f_{w_{0}}$ is a hyperbolic automorphism of $\mathbf{D}$, namely $f_{w_{0}}(z)=\ell^{-1}(\ell(z)+$ $T)$. Otherwise, $f_{w_{0}}$ is a conformal mapping of $\mathbf{D}$ onto $\mathbf{D}$ minus a slit along an analytic Jordan arc $\gamma$ orthogonal to $\partial \mathbf{D}$, with $f_{w_{0}}^{\prime}(1)=1$. Moreover, $f_{w_{0}}=h_{1} \circ p_{\alpha} \circ h_{2}$ for some $h_{1}, h_{2} \in \operatorname{Aut}(\mathbf{D})$ and $\alpha \in(0,1)$ if and only if $w_{0}=\ell^{-1}\left(x_{1}^{0}+\frac{T}{2}+i \arcsin a_{ \pm}(T)\right)$.

Remark 1.1. Note that $z_{0}$ is a boundary point of the value region $\mathcal{V}\left(z_{0}, T\right)$, but does not belong to $\mathcal{V}\left(z_{0}, T\right)$. The proof of the above theorem, given in Section 4, shows that $z_{0}$ would be included, and this would be the only modification of the value region, if we replaced the equality $f^{\prime}(-1)=e^{T}$ in condition (iii) of Theorem 1 by the inequality $f^{\prime}(-1) \leqslant e^{T}$ and removed the requirement $f \neq \mathrm{id}_{\mathbf{D}}$ assuming as a convention that $\mathrm{id}_{\mathbf{D}}$ satisfies (ii). Note also that under the conditions of Theorem 1 modified in this way, $f\left(z_{0}\right)=z_{0}$ if and only if $f=\mathrm{id}_{\mathbf{D}}$, see Remark 2.3.

If $f \in \operatorname{Hol}(\mathbf{D}, \mathbf{D})$ has boundary regular fixed points at \pm 1 , then replacing $f$ by $h \circ f$, where $h$ is a suitable hyperbolic automorphism with the same boundary fixed points, we may suppose that $\tau=1$ is the Denjoy-Wolff point. In this way, as a corollary of Theorems 1 and 2 we easily deduce a sharp estimate for $f^{\prime}(-1) f^{\prime}(1)$, which was obtained earlier with the help of the extremal length method in [15, Section 4]. 
Corollary 1. Let $z_{0} \in \mathbf{D}$ and let $f \in \operatorname{Hol}(\mathbf{D}, \mathbf{D})$ be a univalent function with boundary regular fixed points at 1 and -1 . Then

$$
\sqrt{f^{\prime}(-1) f^{\prime}(1)} \geqslant L\left(\sin \operatorname{Im} \ell\left(z_{0}\right), \sin \operatorname{Im} \ell\left(f\left(z_{0}\right)\right)\right), \quad L(a, b):=\max \left\{\frac{1+a}{1+b}, \frac{1-a}{1-b}\right\} .
$$

Inequality (1.2) is sharp. The equality can occur only for hyperbolic automorphisms and functions of the form $f=h_{1} \circ p_{\alpha} \circ h_{2}, h_{1}, h_{2} \in \operatorname{Aut}(\mathbf{D}), \alpha \in(0,1)$.

Recently, the sharp value regions of $f \mapsto f\left(z_{0}\right)$ have been determined for other classes of univalent self-maps [23, 24, 35, 37]. One of the main instruments is the classical parametric representation of univalent functions, going back to the seminal work by Loewner [29]. In this paper, we use a new variant of Loewner's parametric method, which is specific for functions satisfying conditions of Theorem 1. This variant of parametric representation was discovered quite recently, see [20, 21]. We discuss it in Section 3.

It is also worth mentioning that in [17], using another specific variant of the parametric representation, Goryainov obtained the sharp value region of $f \mapsto f^{\prime}(0)$ in the class of all univalent $f \in \operatorname{Hol}(\mathbf{D}, \mathbf{D}), f(0)=0$, having a boundary regular fixed point at $\sigma=1$ with a given value of $f^{\prime}(1)$.

To complete the Introduction, we recall another related result obtained by Goryainov $[18,19]$. Dropping the univalence requirement, one can study holomorphic selfmaps $f: \mathbf{D} \rightarrow \mathbf{D}$ satisfying conditions (ii) and (iii) in Theorem 1 by using relationships between boundary regular fixed points and the Alexandrov-Clark measures. In particular, according to $[18,19]$, the value region $\mathcal{D}(0, T)$ of $f \mapsto f(0)$ over all such self-maps $f$ is the closed disk whose diameter is the segment $\left[0, \ell^{-1}(T)\right]$, with the boundary point $z_{0}=0$ excluded. Analyzing the functions delivering the boundary points of $\mathcal{D}(0, T)$, one can conclude that $\partial \mathcal{D}(0, T) \bigcap \partial \mathcal{V}(0, T)=\left\{0, \ell^{-1}(T)\right\}$.

\section{Holomorphic self-maps of the unit disk}

In this section we cite some basic theory of holomorphic self-maps of D. More details can be found, e.g., in the monograph [1].

Let $f \in \operatorname{Hol}(\mathbf{D}, \mathbf{D})$ and $\sigma \in \partial \mathbf{D}$. According to the classical Julia-Wolff-Carathéodory Theorem, see, e.g., [1, Theorem 1.2.5, Proposition 1.2.6, Theorem 1.2.7], if

$$
\alpha_{f}(\sigma):=\liminf _{\mathbf{D} \ni z \rightarrow \sigma} \frac{1-|f(z)|}{1-|z|}<+\infty
$$

then

$$
\exists \angle \lim _{z \rightarrow \sigma} f(z)=: f(\sigma) \in \partial \mathbf{D}, \quad \exists \angle \lim _{z \rightarrow \sigma} \frac{f(z)-f(\sigma)}{z-\sigma}=: f^{\prime}(\sigma)=\alpha_{f}(\sigma) \frac{f(\sigma)}{\sigma}
$$

and

$$
\frac{|f(z)-f(\sigma)|^{2}}{1-|f(z)|^{2}} \leqslant\left|f^{\prime}(\sigma)\right| \frac{|z-\sigma|^{2}}{1-|z|^{2}} \quad \text { for all } z \in \mathbf{D},
$$

with the equality sign if and only if $f \in \operatorname{Aut}(\mathbf{D})$. Note that in its turn, existence of the limits in (2.2) satisfying $f(\sigma) \in \partial \mathbf{D}$ and $f^{\prime}(\sigma) \neq \infty$ immediately implies (2.1).

Definition 2.1. Points $\sigma \in \partial \mathbf{D}$ satisfying (2.2) are referred to as regular contact points of $f$. If in addition to (2.2), $f(\sigma)=\sigma$, then $\sigma$ is said to be a regular fixed point of $f$. The number $f^{\prime}(\sigma)$ is called the angular derivative of $f$ at $\sigma$.

Among all fixed points (boundary and internal) of a self-map $f \neq \mathrm{id}_{\mathbf{D}}$, there is one point of special importance for dynamics. On the one hand, if $f(\tau)=\tau$ for 
some $\tau \in \mathbf{D}$, then by the Schwarz Lemma, $\tau$ is the only fixed point of $f$ in $\mathbf{D}$. If in addition, $f$ is not an elliptic automorphism, then $\left|f^{\prime}(\tau)\right|<1$ and hence the sequence of iterates $\left(f^{\circ n}\right), f^{\circ 1}:=f, f^{\circ(n+1)}:=f \circ f^{\circ n}$, converges (to the constant function equal) to $\tau$ locally uniformly in $\mathbf{D}$. On the other hand, if $f$ has no fixed points in $\mathbf{D}$, then by the Denjoy-Wolff Theorem, see, e.g., [1, Theorem 1.2.14, Corollary 1.2.16, Theorem 1.3.9], $f$ has a unique boundary regular fixed point $\tau \in \partial \mathbf{D}$ such that $f^{\prime}(\tau) \leqslant 1$ and moreover, $f^{\circ n} \rightarrow \tau$ locally uniformly in $\mathbf{D}$ as $n \rightarrow+\infty$.

Definition 2.2. The point $\tau$ above is referred to as the Denjoy-Wolff point of $f$.

Remark 2.3. Since the strict inequality holds in (2.3) unless $f \in \operatorname{Aut}(\mathbf{D})$, a self-map $f$ can have a fixed point in $\mathbf{D}$ and a boundary regular fixed point $\sigma$ with $f^{\prime}(\sigma) \leqslant 1$ only if $f=\mathrm{id}_{\mathbf{D}}$.
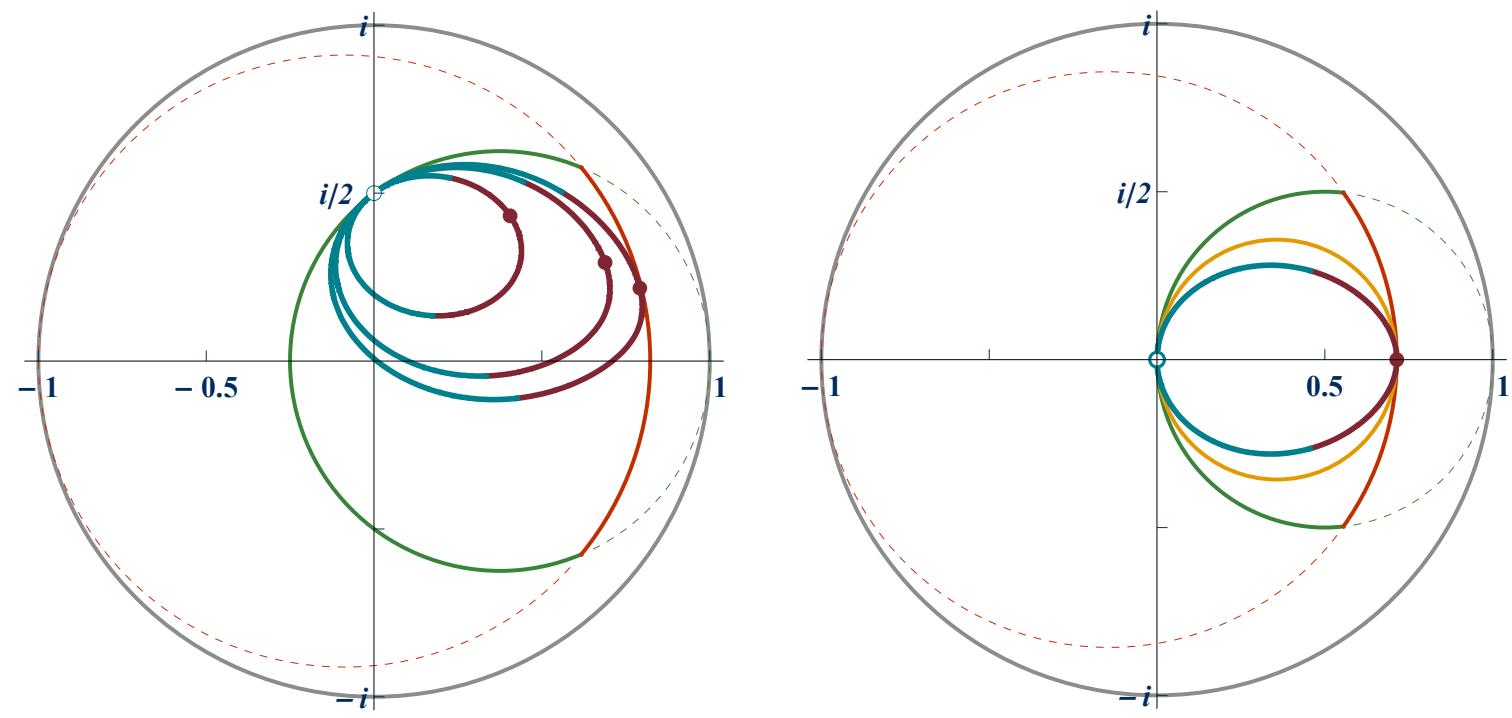

$-\partial \mathbf{D} \circ z_{0}-\Gamma^{-} \cdot \ell^{-1}\left(\ell\left(z_{0}\right)+T\right)$

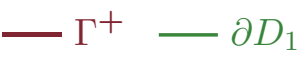

$\partial D_{2}$ $\partial \mathcal{D}(0, T)$

Figure 1. The value region $\mathcal{V}\left(z_{0}, T\right)$ and the disks $D_{1}, D_{2}$ for $z_{0}:=i / 2, T \in\{\log 2, \log 4, \log 6\}$ and for $z_{0}:=0, T:=\log 6$. The right picture also shows the disk $\mathcal{D}(0, T)$. Notation $\Gamma^{ \pm}$is explained in Section 4.

Remark 2.4. Let $f_{n}(z):=-\sigma H^{-1}(\alpha / H(z / \sigma)+\beta /(H(z / \sigma)+n))$, where $n \in \mathbf{N}$, $\alpha, \beta>0$, and $H(z):=(1+z) /(1-z)$. Note that $f_{n}(\mathbf{D}) \subset \mathbf{D}$ for all $n \in \mathbf{N}$ and that $f_{n}(z) \longrightarrow f(z):=(z+c) /(1+\bar{c} z), c:=\sigma(1-\alpha) /(1+\alpha)$, locally uniformly in $\mathbf{D}$ as $n \rightarrow+\infty$. Moreover, $f_{n}(\sigma)=f(\sigma)=\sigma$ and $f_{n}^{\prime}(\sigma)=\alpha+\beta$ for all $n \in \mathbf{N}$, but $f^{\prime}(\sigma)=\alpha$. This example shows that the map $f \mapsto f^{\prime}(\sigma)$ is not continuous. However, it turns out to be semicontinuous in the following sense. Suppose that $f_{n}(z) \rightarrow f(z)$ as $n \rightarrow+\infty$ and that $\sigma \in \partial \mathbf{D}$ is a boundary regular fixed point of $f_{n} \in \operatorname{Hol}(\mathbf{D}, \mathbf{D})$ for all $n \in \mathbf{N}$ with $\alpha:=\liminf _{n \rightarrow+\infty} f_{n}^{\prime}(\sigma)<+\infty$. Then passing in Julia's inequality (2.3) applied for functions $f_{n}$ to the limit, we conclude that $f$ satisfies (2.3) with $\left|f^{\prime}(\sigma)\right|$ replaced by $\alpha$. It follows that $\alpha_{f}(\sigma) \leqslant \alpha<+\infty$. Therefore, either $f \equiv \sigma$ or $f \in \operatorname{Hol}(\mathbf{D}, \mathbf{D})$ and $\sigma$ is a regular boundary fixed point of $f$ with $f^{\prime}(\sigma) \leqslant \alpha$. As a consequence, the set of all $f \in \operatorname{Hol}(\mathbf{D}, \mathbf{D})$ sharing two different boundary regular fixed points $\sigma_{1}$ and $\sigma_{2}$ and satisfying $f^{\prime}\left(\sigma_{j}\right) \leqslant \alpha_{j}<+\infty, j=1,2$, is compact. 
According to inequality (2.3), the value region $\mathcal{V}\left(z_{0}, T\right)$ in Theorem 1 lies in the intersection of two closed disks $D_{1}, D_{2} \subset \overline{\mathbf{D}}$ whose boundaries pass through $z_{0}$ and $\tau=1$ and through $\ell^{-1}\left(\ell\left(z_{0}\right)+T\right)$ and $\sigma=-1$, respectively. Comparison of $\mathcal{V}\left(z_{0}, T\right)$ with $D_{1} \cap D_{2}$ is show in Figure 1. On the right picture, for which $z_{0}=0$, we also place the value range $\mathcal{D}(0, T)$ of $f \mapsto f(0)$ over all holomorphic but not necessary injective maps $f: \mathbf{D} \rightarrow \mathbf{D}$ satisfying conditions (ii) and (iii) in Theorem 1 , see $[18,19]$.

\section{Parametric representation}

Denote the class of all $f \in \operatorname{Hol}(\mathbf{D}, \mathbf{D})$ satisfying conditions (i)-(iii) in Theorem 1 by $\mathfrak{U}(T)$. The following theorem, proved in [21], gives a parametric representation for $\mathfrak{U}(T)$ in terms of a Loewner-Kufarev-type ODE.

Theorem 3. [21, Corollary 1.2] The class $\mathfrak{U}(T)$ coincides with the set of all functions representable in the form $f(z)=w_{z}(T)$ for all $z \in \mathbf{D}$, where $w_{z}(t)$ is the unique solution to the initial value problem

$$
\frac{\mathrm{d} w_{z}}{\mathrm{~d} t}=\frac{1}{4}\left(1-w_{z}\right)^{2}\left(1+w_{z}\right) q\left(w_{z}, t\right), \quad t \in[0, T], \quad w_{z}(0)=z
$$

with some function $q: \mathbf{D} \times[0, T] \rightarrow \mathbf{C}$ satisfying the following conditions:

(i) for every $z \in \mathbf{D}, q(z, \cdot)$ is measurable on $[0, T]$;

(ii) for a.e. $t \in[0, T], q(\cdot, t)$ has the following integral representation

$$
q(z, t)=\int_{\partial \mathbf{D} \backslash\{1\}} \frac{1-\kappa}{1+\kappa z} \mathrm{~d} \nu_{t}(\kappa)
$$

where $\nu_{t}$ is a probability measure on $\partial \mathbf{D} \backslash\{1\}$.

Remark 3.1. A related parametric representation for a class of univalent selfmaps of a strip was considered in [13].

Remark 3.2. In many cases, it is more convenient to deal with the the union $\mathfrak{U}^{\prime}(T):=\bigcup_{0 \leqslant T^{\prime} \leqslant T} \mathfrak{U}\left(T^{\prime}\right)$, where we define $\mathfrak{U}(0):=\left\{\operatorname{id}_{\mathbf{D}}\right\}$. Indeed, it is evident from the argument of Remark 2.4 that in contrast to $\mathfrak{U}(T)$, the class $\mathfrak{U}^{\prime}(T)$ is compact. Moreover, it is easy to see that Theorem 3 gives representation of $\mathfrak{U}^{\prime}(T)$ if all probability measures $\nu_{t}$ in (3.2) are replaced with all positive Borel measures $\nu_{t}$ satisfying

$$
\nu_{t}(\partial \mathbf{D} \backslash\{1\}) \in[0,1] .
$$

Note that the possibility of $\nu_{t}=0$ is not excluded.

Remark 3.3. Obviously, the right-hand side of (3.1) can be written as $G\left(w_{z}, t\right)$, where $G(z, t):=\frac{1}{4}(1-z)^{2}(1+z) q(z, t)$ with $q$ satisfying conditions (i) and (ii) in Theorem 3. By [20, Theorem 1], $G(\cdot, t)$ is an infinitesimal generator in $\mathbf{D}$ for each $t \in[0, T]$. For simplicity, extend $G$ to all $t \geqslant 0$ by setting $G(z, t) \equiv 0$ for any $t>T$. Then according to the general theory of Loewner-Kufarev-type equations, see [4, Sections 3-5], for any $s \geqslant 0$ and any $z \in \mathbf{D}$, the initial value problem $\mathrm{d} w / \mathrm{d} t=$ $G(w, t), t \geqslant s, w(s)=z$, has a unique solution $w=w_{z, s}(t)$ defined for all $t \geqslant s$ and the functions $\varphi_{s, t}(z):=w_{z, s}(t), z \in \mathbf{D}, t \geqslant s \geqslant 0$, form an evolution family, see [4, Definition 3.1].

Proposition 1. Let $\vartheta:[0, T] \rightarrow(-\pi, \pi) \backslash\{0\}, T>0$, be a $C^{1}$-smooth function. Suppose that in the conditions of Theorem $3, \mathrm{~d} \nu_{t}\left(e^{i \theta}\right)=\delta(\theta-\vartheta(t)) \mathrm{d} \theta$ for all $t \in[0, T]$, where $\delta$ stands for the Dirac delta function. Then $f$ maps $\mathbf{D}$ onto $\mathbf{D} \backslash \gamma$, where $\gamma$ is a slit in $\mathbf{D}$, i.e. $\gamma$ is the image of a homeomorphism $\gamma:[0,1] \mapsto \overline{\mathbf{D}}$ with $\gamma([0,1)) \subset \mathbf{D}$ and $\gamma(1) \in \partial \mathbf{D}$. Moreover, 
(i) if $\vartheta$ is a real-analytic function on $[0, T]$, then $\gamma$ is a real-analytic Jordan arc orthogonal to $\mathbf{D}$;

(ii) $\gamma$ is a circular arc or a straight line segment orthogonal to $\partial \mathbf{D}$ if and only if

$$
\lambda(t):=i \frac{1+e^{i \vartheta(t)}}{1-e^{i \vartheta(t)}}=C_{1} e^{-t / 2}\left(C_{2} e^{t / 2}+\sqrt{C_{2}^{2}\left(e^{t}-1\right)+1}\right)^{3}
$$

for all $t \in[0, T]$ and some constants $C_{1}, C_{2} \in \mathbf{R}, C_{1} \neq 0$.

Proof. In the conditions of the proposition, (3.1) takes the following form:

$$
\frac{\mathrm{d} w_{z}}{\mathrm{~d} t}=\frac{1}{4}\left(1-w_{z}\right)^{2}\left(1+w_{z}\right) \frac{1-e^{i \vartheta(t)}}{1+e^{i \vartheta(t)} w_{z}}, \quad t \in[0, T], \quad w_{z}(0)=z .
$$

The change of variables $\omega_{z}:=H\left(w_{z}\right)$, where $H(w):=i(1+w) /(1-w)$ maps $\mathbf{D}$ conformally onto $\mathbf{H}:=\{\omega: \operatorname{Im} \omega>0\}$, transforms the above problem to

$$
\frac{\mathrm{d} \omega_{z}}{\mathrm{~d} t}=\frac{\omega_{z}}{1-\lambda(t) \omega_{z}}, \quad t \in[0, T], \quad \omega_{z}(0)=H(z)
$$

where $\lambda(t):=H\left(e^{i \vartheta(t)}\right)$ for all $t \in[0, T]$. Making further change of variables

$$
\hat{\omega}_{z}(t):=\omega_{z}(t)+\int_{0}^{t} \frac{\mathrm{d} s}{\lambda(s)}, \quad \xi(t):=\frac{1}{\lambda(t)}+\int_{0}^{t} \frac{\mathrm{d} s}{\lambda(s)}, \quad \tau=v(t):=\frac{1}{2} \int_{0}^{t} \frac{\mathrm{d} s}{\lambda(s)^{2}},
$$

we obtain the chordal Loewner equation

$$
\frac{\mathrm{d} \hat{\omega}_{z}}{\mathrm{~d} \tau}=\frac{2}{\xi-\hat{\omega}_{z}}, \quad \tau \in[0, v(T)], \quad \hat{\omega}_{z}(0)=H(z) .
$$

The geometry of solutions to (3.7) is well-studied, see, e.g., [27, 30, 36, 22, 39]; see also [25]. In particular, since the function $s \mapsto \xi\left(v^{-1}(s)\right)$ is $C^{1}$-smooth, it follows that $z \mapsto \hat{\omega}_{z}(T)$ maps $\mathbf{D}$ onto $\mathbf{H}$ minus a slit along some Jordan arc $\gamma_{0}$. Taking into account that $w_{z}(T)=H^{-1}\left(\hat{\omega}_{z}(T)-C\right)$, where $C:=\int_{0}^{T} \lambda(t)^{-1} \mathrm{~d} t$, this proves the first part of the proposition.

If $\vartheta$ is real-analytic, then $s \mapsto \xi\left(v^{-1}(s)\right)$ is real-analytic on $[0, T]$ as well, and hence by [28, Theorem 1.4], $\gamma_{0}$ is a real-analytic Jordan arc. Moreover, the argument of [28, Section 6.1] shows that in such a case, $\gamma_{0}$ is orthogonal to $\mathbf{R}$. This proves (i).

It remains to prove (ii). Suppose that $\gamma$ is a circular arc or a straight line segment orthogonal to $\partial \mathbf{D}$. Then we can find a linear-fractional transformation $H_{*}$ of $\mathbf{D}$ onto $\mathbf{H}$ such that $H_{*}(\gamma)=[0, i]$. Let $\left(\varphi_{s, t}\right)$ be the evolution family associated with equation (3.5), see Remark 3.3. Note that $\varphi_{t, T}(\mathbf{D}) \supset \varphi_{t, T}\left(\varphi_{0, t}(\mathbf{D})\right)=$ $\varphi_{0, T}(\mathbf{D})=f(\mathbf{D})$ for any $t \in[0, T]$. It follows that the intersection of a sufficiently small neighbourhood of $H_{*}^{-1}(\infty)$ with $\partial \varphi_{t, T}(\mathbf{D})$ is an open arc of $\partial \mathbf{D}$ containing $H_{*}^{-1}(\infty)$. Therefore, for each $t \in[0, T]$, there exists a unique $h_{t} \in \operatorname{Aut}(\mathbf{D})$ such that $g_{t}:=H_{*} \circ \varphi_{t, T} \circ h_{t} \circ H_{*}^{-1} \in \operatorname{Hol}(\mathbf{H}, \mathbf{H})$ satisfies the Laurent expansion $g_{t}(z)=z-c(t) / z+\ldots$ at $\infty$ with some $c(t) \in \mathbf{R}$.

Denote $H_{t}:=H_{*} \circ h_{t}^{-1}$ for all $t \in[0, T]$. By construction, $\mathbf{H} \backslash[0, i]=g_{0}(\mathbf{H}) \subset$ $g_{t}(\mathbf{H}) \subset g_{T}(\mathbf{H})=\mathbf{H}$ for all $t \in[0, T]$. Thanks to continuity of $\vartheta$, the function $t \mapsto c(t)$ is $C^{1}$-smooth. Therefore, according to the classical result [26] by Kufarev et al, see also [12], for any $z \in \mathbf{D}, \tilde{\omega}_{z}(t):=g_{t}^{-1} \circ g_{0}\left(H_{0}(z)\right), t \in[0, T]$, is the unique solution to the initial value problem $\mathrm{d} \tilde{\omega}_{z} / \mathrm{d} t=-c^{\prime}(t) / \tilde{\omega}_{z}, \tilde{\omega}_{z}(0)=H_{0}(z) \in \mathbf{H}$. 
By construction, $\tilde{\omega}_{z}(t)=H_{t}\left(w_{z}\right)$ for all $t \in[0, T]$ and all $z \in \mathbf{D}$. Comparing the differential equations for $\tilde{\omega}_{z}$ and $w_{z}$, one can conclude that for all $t \in[0, T]$,

$$
H_{t}(w):=\frac{\lambda(t) H(w)-1}{a(t)(\lambda(t) H(w)-1)+b(t)}
$$

with real coefficients $a(t)$ and $b(t)$ satisfying

$$
\mathrm{d} a / \mathrm{d} t=a^{3} / b^{2}, \quad \mathrm{~d} b / \mathrm{d} t=-3 a+b+3 a^{2} / b, \quad t \in[0, T],
$$

and such that $\lambda^{\prime}(t) / \lambda(t)=1-3 a(t) / b(t)$ and $b(t) \lambda(t)>0$ for all $t \in[0, T]$. System (3.9) can be solved by introducing a new unknown function $k(t):=a(t) / b(t)$. In this way, one can easily check that $\lambda$ must be of the form (3.4).

Conversely, if $\lambda$ is given by (3.4), then system (3.9) has a real-valued solution satisfying $\lambda^{\prime}(t) / \lambda(t)=1-3 a(t) / b(t)$ and $b(t) \lambda(t)>0$ for all $t \in[0, T]$. It follows that for any $z \in \mathbf{D}$, the function $\tilde{\omega}_{z}(t):=H_{t}\left(w_{z}(t)\right)$, where $H_{t}$ is given by (3.8), is a solution to $\mathrm{d} \tilde{\omega}_{z} / \mathrm{d} t=-1 /\left(b(t)^{2} \tilde{\omega}_{z}\right), t \in[0, T], \tilde{\omega}_{z}(0)=H_{0}(z) \in \mathbf{H}$. Solving the latter initial value problem for $\tilde{\omega}_{z}$, we conclude that the image of the map $\mathbf{D} \ni z \mapsto \tilde{\omega}_{z}(T)$ is the domain $\mathbf{H} \backslash\left[0, i \sqrt{Q_{T}}\right], Q_{T}:=2 \int_{0}^{T} b(t)^{-2} \mathrm{~d} t$. Thus, $\gamma=H_{T}^{-1}\left(\left[0, i \sqrt{Q_{T}}\right]\right)$ is a circular arc or a straight line segment orthogonal to $\partial \mathbf{D}$. The proof is now complete.

\section{Proof of the main results}

In this section we prove Theorems 1 and 2. Fix $T>0$. We start by considering the problem to determine the compact value region $\left\{f\left(z_{0}\right): f \in \mathfrak{U}^{\prime}(T)\right\}$. Thanks to Theorem 3 and Remark 3.2, it coincides with the reachable set $\left\{w_{z_{0}}(T)\right\}$ of the controllable system (3.1) in which the measure-valued control $t \mapsto \nu_{t}$ satisfies (3.3). The change of variables

$$
\zeta=\ell(w), \quad \lambda=i \frac{1+\kappa}{1-\kappa}
$$

reduces our problem to finding the reachable set $\Omega_{T}^{\prime}:=\{\zeta(T)\}$ for the following controllable system

$$
\frac{\mathrm{d} \zeta}{\mathrm{d} t}=\int_{\mathbf{R}} \frac{\mathrm{d} \mu_{t}(\lambda)}{1-i \lambda e^{\zeta}}, \quad t \in[0, T] ;\left.\quad \zeta\right|_{t=0}=\zeta_{0}:=\ell\left(z_{0}\right)
$$

where $\mu_{t}$ 's are positive Borel measures on $\mathbf{R}$ with $\mu_{t}(\mathbf{R}) \leqslant 1$. By using the prime in the notation $\Omega_{T}^{\prime}$ we emphasize that this reachable set corresponds to the class $\mathfrak{U}^{\prime}(T)$.

Denote $x_{1}:=\operatorname{Re} \zeta$ and $x_{2}:=\operatorname{Im} \zeta$. Note that $x_{2} \in\left(-\frac{\pi}{2}, \frac{\pi}{2}\right)$. For any fixed $\zeta=x_{1}+i x_{2} \in \mathbf{S}$, the range of the right-hand side in (4.1), regarded as a function of the measure $\mu_{t}$, is the disk

$$
\left\{\omega \in \mathbf{C}:\left|\omega-\frac{e^{-i x_{2}}}{2 \cos x_{2}}\right| \leqslant \frac{1}{2 \cos x_{2}}\right\} .
$$

Therefore, replacing the measure-valued control $t \mapsto \mu_{t}$ with the complex-valued control

$$
u(t):=2 e^{i x_{2}} \cos x_{2} \int_{\mathbf{R}} \frac{\mathrm{d} \mu_{t}(\lambda)}{1-i \lambda e^{x_{1}+i x_{2}}},
$$


we can rewrite (4.1) in the following form

$$
\begin{aligned}
& \frac{\mathrm{d} x_{1}}{\mathrm{~d} t}=\operatorname{Re} \frac{u(t) e^{-i x_{2}}}{2 \cos x_{2}}=\frac{1}{2} \operatorname{Re} u(t)+\frac{\operatorname{tg} x_{2}}{2} \operatorname{Im} u(t), \quad x_{1}(0)=x_{1}^{0}:=\operatorname{Re} \zeta_{0}, \\
& \frac{\mathrm{d} x_{2}}{\mathrm{~d} t}=\operatorname{Im} \frac{u(t) e^{-i x_{2}}}{2 \cos x_{2}}=\frac{1}{2} \operatorname{Im} u(t)-\frac{\operatorname{tg} x_{2}}{2} \operatorname{Re} u(t), \quad x_{2}(0)=x_{2}^{0}:=\operatorname{Re} \zeta_{0},
\end{aligned}
$$

where $u:[0, T] \rightarrow U:=\{u:|u-1| \leqslant 1\}$ is an arbitrary measurable function.

Introduce the Hamilton function

$$
\mathcal{H}\left(x_{1}, x_{2}, \Psi_{1}, \Psi_{2}, u\right):=\Psi_{1} \operatorname{Re} \frac{u e^{-i x_{2}}}{2 \cos x_{2}}+\Psi_{2} \operatorname{Im} \frac{u e^{-i x_{2}}}{2 \cos x_{2}}=\operatorname{Re} \frac{u e^{-i x_{2}}\left(\Psi_{1}-i \Psi_{2}\right)}{2 \cos x_{2}},
$$

where $\Psi_{1}, \Psi_{2}$ satisfy the adjoint system of ODEs

$$
\frac{\mathrm{d} \Psi_{1}}{\mathrm{~d} t}=-\frac{\partial \mathcal{H}}{\partial x_{1}}=0, \quad \frac{\mathrm{d} \Psi_{2}}{\mathrm{~d} t}=-\frac{\partial \mathcal{H}}{\partial x_{2}}=-\operatorname{Im} \frac{u(t)\left(\Psi_{1}-i \Psi_{2}\right)}{2 \cos ^{2} x_{2}} .
$$

Boundary points of the reachable set $\Omega_{T}^{\prime}$, forming a dense subset of $\partial \Omega_{T}^{\prime}$, are generated by the driving functions $u^{*}$ satisfying the necessary optimal condition in the form of Pontryagin's maximum principle,

$$
\max _{u \in U} \mathcal{H}\left(x_{1}(t), x_{2}(t), \Psi_{1}(t), \Psi_{2}(t), u\right)=\mathcal{H}\left(x_{1}(t), x_{2}(t), \Psi_{1}(t), \Psi_{2}(t), u^{*}(t)\right)
$$

for all $t \in[0, T]$, see, e.g., [34]. Trajectories $\left(x_{1}(t), x_{2}(t)\right)$ in (4.5) are optimal in the reachable set problem, and $\left(\Psi_{1}(t), \Psi_{2}(t)\right)$ satisfy the adjoint system (4.4) with the optimal trajectories. In particular, $\left(\Psi_{1}(t), \Psi_{2}(t)\right)$ does not vanish, and hence the maximum in (4.5) is attained at the unique point $u^{*}=1+e^{i\left(x_{2}+\varphi\right)}$, where $\varphi:=\arg \left(\Psi_{1}+i \Psi_{2}\right)$. Therefore, from (4.2)-(4.4) for the optimal trajectories we obtain

$$
\begin{aligned}
\frac{\mathrm{d} x_{1}}{\mathrm{~d} t} & =\frac{\cos \varphi+\cos x_{2}}{2 \cos x_{2}}, \quad x_{1}(0)=x_{1}^{0}, \\
\frac{\mathrm{d} x_{2}}{\mathrm{~d} t} & =\frac{\sin \varphi-\sin x_{2}}{2 \cos x_{2}}, \quad x_{2}(0)=x_{2}^{0}, \\
\frac{\mathrm{d} \Psi_{1}}{\mathrm{~d} t} & =0, \\
\frac{\mathrm{d} \Psi_{2}}{\mathrm{~d} t} & =\frac{\sin \varphi-\sin x_{2}}{2 \cos ^{2} x_{2}}\left|\Psi_{1}-i \Psi_{2}\right| .
\end{aligned}
$$

System (4.6)-(4.9) is invariant w.r.t. multiplication of $\left(\Psi_{1}, \Psi_{2}\right)$ by a positive constant. Therefore, we may assume that either $\Psi_{1} \equiv 0$, or $\Psi_{1} \equiv 1$, or $\Psi_{1} \equiv-1$.

If $\Psi_{1} \equiv 0$, then $\varphi= \pm \pi / 2$ and we easily get that for all $t \geqslant 0$,

$$
x_{1}(t)=x_{1}(0)+t / 2, \quad \sin x_{2}(t)=a_{ \pm}(t):=e^{-t / 2} \sin x_{2}(0) \pm\left(1-e^{-t / 2}\right) .
$$

Now let $\Psi_{1} \equiv 1$. Then $\varphi \in(-\pi / 2, \pi / 2)$ and equation (4.9) takes the following form

$$
\frac{\mathrm{d} \varphi}{\mathrm{d} t}=\frac{\sin \varphi-\sin x_{2}}{2 \cos ^{2} x_{2}} \cos \varphi=\frac{\cos \varphi}{\cos x_{2}} \frac{\mathrm{d} x_{2}}{\mathrm{~d} t} .
$$

System (4.7), (4.11) admits the first integral

$$
I\left(x_{2}, \varphi\right):=\frac{1-\sin \varphi}{1+\sin \varphi} \frac{1+\sin x_{2}}{1-\sin x_{2}}>0,
$$


and as a result it can be integrated in quadratures. Namely, if $C:=I\left(x_{2}(0), \varphi(0)\right)$ $\neq 1$, we obtain the following identities

$$
\begin{aligned}
B_{1}(t)-C B_{2}(t) & =(C-1) t / 2, \\
x_{1}(t)-x_{1}(0) & =\frac{B_{1}(t)-\sqrt{C} B_{2}(t)}{\sqrt{C}-1},
\end{aligned}
$$

where

$$
B_{1}(t):=\log \frac{1-\sin x_{2}(t)}{1-\sin x_{2}(0)}, \quad B_{2}(t):=\log \frac{1+\sin x_{2}(t)}{1+\sin x_{2}(0)} .
$$

Excluding $C$ from (4.12), (4.13) and setting $t=T$ gives

$$
\begin{aligned}
x_{1}(T) & =x_{1}(0)+\frac{1}{2}\left(T+\sqrt{\left(T+2 B_{1}(T)\right)\left(T+2 B_{2}(T)\right)}\right) \\
& =x_{1}(0)+\frac{T}{2}+\sqrt{R\left(\sin x_{2}(T), T\right)},
\end{aligned}
$$

where we took into account that according to (4.12),

$$
\frac{\mathrm{d}}{\mathrm{d} t}\left(t+2 B_{1}(t)\right)=\frac{2 C}{1+\sin x_{2}(t)+C\left(1-\sin x_{2}(t)\right)}>0
$$

and therefore, $T+2 B_{1}(T)>0$.

For $C=1$, we have $\varphi(t)=x_{2}(t)$ and hence $\mathrm{d} \varphi / \mathrm{d} t=\mathrm{d} x_{2} / \mathrm{d} t=0, \mathrm{~d} x_{1} / \mathrm{d} t=1$. Therefore, if $C=1$, then (4.12) and (4.14) hold as well. Since $C>0$, from (4.12) we obtain that $x_{2}(T) \in J(T):=\left(\arcsin a_{-}(T), \arcsin a_{+}(T)\right)$. On the other hand, for any $x \in J(T)$ there exists a unique $C=C(x)>0$ that verifies (4.12) with $T$ and $x$ substituted for $t$ and $x_{2}(t)$, respectively. Solving $I\left(x_{2}(0), \varphi(0)\right)=C(x)$ provides us with the initial condition in equation (4.11) for which $x_{2}(T)=x$.

Investigating the case $\Psi_{1} \equiv-1$ in a similar way, we conclude that $\partial \Omega_{T}^{\prime}$ is the union of the two Jordan arcs

$$
\Gamma^{ \pm}(T):=\left\{x_{1}+i x_{2} \in \mathbf{S}: a_{-}(T) \leqslant \sin x_{2} \leqslant a_{+}(T), x_{1}=x_{1}^{0}+\frac{T}{2} \pm \sqrt{R\left(\sin x_{2}, T\right)}\right\},
$$

which do not intersect except for the common end-points $\omega^{ \pm}:=x_{1}^{0}+T / 2+$ $i \arcsin a_{ \pm}(T)$, delivered by solutions (4.10). Taking into account that by the very definition, $\mathfrak{U}^{\prime}\left(T^{\prime}\right) \subset \mathfrak{U}^{\prime}(T)$ for any $T^{\prime} \in[0, T]$, it follows that $\Omega_{T}^{\prime}=V\left(\zeta_{0}, T\right)$.

The next step in the proof is to pass from the class $\mathfrak{U}^{\prime}(T)$ to the class $\mathfrak{U}(T)$. In the problem of finding the value region of the functional $f \mapsto f\left(z_{0}\right)$, this is equivalent to replacing the range $U$ of the admissible controls $u$ in (4.2)-(4.3) by $U \backslash\{0\}$. Denote by $\Omega_{T}$ the corresponding reachable set. By re-scaling the time, the problem to find $\Omega_{T^{\prime}}, T^{\prime} \in(0, T)$, can be restated as the reachable set problem at the same time $T$ and for the same controllable system, but with the value range of admissible controls restricted to $\alpha(U \backslash\{0\}), \alpha:=T^{\prime} / T$. Note also that $\Gamma^{+}(T) \cup \Gamma^{-}(T) \backslash\left\{\zeta_{0}\right\} \subset \Omega_{T}$ for any $T>0$. Since $\alpha(U \backslash\{0\}) \subset U \backslash\{0\}$ for any $\alpha \in(0,1)$, it follows that

$$
\Gamma^{+}\left(T^{\prime}\right) \cup \Gamma^{-}\left(T^{\prime}\right) \backslash\left\{\zeta_{0}\right\} \subset \Omega_{T^{\prime}} \subset \Omega_{T} \quad \text { for any } T \in(0, T] .
$$

Thus $\Omega_{T}=V\left(\zeta_{0}, T\right) \backslash\left\{\zeta_{0}\right\}$, which completes the proof of Theorem 1 .

To prove Theorem 2, we have to identify the functions delivering the boundary points of $\mathcal{V}\left(z_{0}, T\right)$. They correspond to the controls $u^{*}$ satisfying Pontryagin's maximum principle (4.5). It is easy to see from the above argument that every point $\omega \in \partial \Omega_{T}^{\prime} \backslash\left\{\zeta_{0}\right\}$ corresponds to a unique control, which is $C^{1}$-smooth and takes values on $\partial U \backslash\{0\}$. It follows that the corresponding measures $\mu_{t}$ in (4.1) and the measures 
$\nu_{t}$ in the Loewner-type representation (3.1), (3.2) are also unique. They are probability measures concentrated at one point that moves smoothly with $t$. Namely, $\mathrm{d} \mu_{t}(\lambda)=\delta\left(\lambda-\lambda^{*}(t)\right) \mathrm{d} \lambda$, where

$$
\lambda^{*}(t):=\frac{1-2 \cos x_{2}(t) /\left(e^{-i x_{2}(t)}+e^{i \varphi(t)}\right)}{i e^{x_{1}(t)+i x_{2}(t)}}=e^{-x_{1}(t)} \frac{\sin \frac{\varphi(t)-x_{2}(t)}{2}}{\cos \frac{\varphi(t)+x_{2}(t)}{2}} .
$$

The point $\omega=\omega_{0}:=\zeta_{0}+T \in \Gamma^{+}$corresponds to $C=1$, in which case $\varphi(t)=$ $x_{2}(t)$ for all $t \in[0, T]$ and hence $\lambda^{*}(t) \equiv 0$. Therefore, from (4.1) we see that the unique $f \in \mathfrak{U}(T)$ delivering the boundary point $\ell^{-1}\left(\omega_{0}\right)$ of $\mathcal{V}\left(z_{0}, T\right)$ is the hyperbolic automorphism

$$
f(z)=\frac{z+c(T)}{1+c(T) z}, \quad c(T):=\frac{e^{T}-1}{e^{T}+1}, \quad \text { for all } z \in \mathbf{D} .
$$

For the common end-points $\omega^{ \pm}$of $\Gamma^{+}$and $\Gamma^{-}$, which correspond to $\varphi= \pm \pi / 2$, formula (4.15) simplifies to $\lambda^{*}(t)= \pm e^{-x_{1}(t)}$. In view of (4.10), the latter expression coincides with $\lambda(t)$ given by (3.4) if we set $C_{1}:= \pm e^{-x_{1}^{0}}$ and $C_{2}:=0$. Taking into account the correspondence between $\mu_{t}$ and $\nu_{t}$ and applying Proposition 1, we conclude that the unique functions $f \in \mathfrak{U}(T)$ delivering the points $\ell^{-1}\left(\omega^{ \pm}\right)$map $\mathbf{D}$ onto $\mathbf{D}$ minus a slit along a circular arc or a segment of a straight line orthogonal to $\partial \mathbf{D}$.

It remains to compare $\lambda^{*}(t)$ given by (4.15) with $\lambda(t)$ given by (3.4) for the case $\omega \in \partial \Omega_{T} \backslash\left\{\zeta_{0}, \omega_{0}, \omega^{+}, \omega^{-}\right\}$. Suppose $\omega \in \Gamma^{+} \backslash\left\{\omega_{0}, \omega^{+}, \omega^{-}\right\}$. Using equations (4.6), (4.7), (4.11) and taking into account the first integral $I\left(x_{2}, \varphi\right)=C$, we find that

$$
\left(1+2 \frac{\mathrm{d}}{\mathrm{d} t} \log \lambda^{*}(t)\right)^{2}=\left(\frac{\cos \varphi(t)}{\cos x_{2}(t)}\right)^{2}=\frac{C\left(1-a^{2}\right)}{\left((1+C) a+(1-C) a^{2}\right)^{2}}, \quad a:=\sin x_{2}(t),
$$

while $(1+2(\mathrm{~d} / \mathrm{d} t) \log \lambda(t))^{2}=9 C_{2}^{2} e^{t} /\left(1+C_{2}^{2}\left(e^{t}-1\right)\right)$. However, according to (4.12), $e^{t}$ cannot be expressed as a rational function of $\sin x_{2}(t)$. This shows that $\lambda^{*}$ is not of the form (3.4) and hence, by Proposition 1, the unique function $f \in \mathfrak{U}(T)$ that delivers the boundary point $\ell^{-1}(\omega)$ maps $\mathbf{D}$ onto $\mathbf{D}$ minus a slit along a real-analytic arc $\gamma$ orthogonal to $\partial \mathbf{D}$ but different from a circular arc or a segment of a straight line. A similar argument applied to the case $\omega \in \Gamma^{-} \backslash\left\{\zeta_{0}, \omega^{+}, \omega^{-}\right\}$completes the proof of Theorem 2 .

\section{References}

[1] Abate, M.: Iteration theory of holomorphic maps on taut manifolds. - Research and Lecture Notes in Mathematics, Complex Analysis and Geometry, Mediterranean, Rende, 1989.

[2] Anderson, J. M., and A. VAsil'ev: Lower Schwarz-Pick estimates and angular derivatives. - Ann. Acad. Sci. Fenn. Math. 33:1, 2008, 101-110.

[3] Bolotnikov, V., M. Elin, and D. Shoikhet: Inequalities for angular derivatives and boundary interpolation. - Anal. Math. Phys. 3:1, 2013, , 63-96.

[4] Bracci, F., M. Contreras, and S. Díaz-Madrigal: Evolution families and the Loewner equation I: the unit disc. - J. Reine Angew. Math. 672, 2012, 1-37.

[5] Bracci, F., M. D. Contreras, S. Díaz-Madrigal, and P. Gumenyuk: Boundary regular fixed points in Loewner theory. - Ann. Mat. Pura Appl. (4) 194:1, 2015, 221-245.

[6] Bracci, F., and P. Gumenyuk: Contact points and fractional singularities for semigroups of holomorphic self-maps of the unit disc. - J. Anal. Math. 130:1, 2016, 185-217. 
[7] Contreras, M. D., and S. Díaz-Madrigal: Analytic flows on the unit disk: angular derivatives and boundary fixed points. - Pacific J. Math. 222, 2005, 253-286.

[8] Contreras, M. D., S. Díaz-Madrigal, and Ch. Pommerenke: Fixed points and boundary behaviour of the Koenigs function. - Ann. Acad. Sci. Fenn. Math. 29:2, 2004, 471-488.

[9] Contreras, M. D., S. Díaz-Madrigal, and Ch. Pommerenke: On boundary critical points for semigroups of analytic functions. - Math. Scand. 98:1, 2006, 125-142.

[10] Contreras, M. D., S. Díaz-Madrigal, and A. Vasil'Ev: Digons and angular derivatives of analytic self-maps of the unit disk. - Complex Var. Elliptic Equ. 52:8, 2007, 685-691.

[11] Cowen, C. C., and C. Pommerenke: Inequalities for the angular derivative of an analytic function in the unit disk. - J. London Math. Soc. (2) 26:2, 1982, 271-289.

[12] Del Monaco, A., and P. Gumenyuk: Chordal Loewner equation. - In: Complex analysis and dynamical systems VI, Part 2, Contemp. Math. 667, Israel Math. Conf. Proc., Amer. Math. Soc., Providence, RI, 2016, 63-77.

[13] Dubovikov, D. A.: An analog of the Löwner equation for mappings of strips. - Izv. Vyssh. Uchebn. Zaved. Mat. 2007:8, 2007, 77-80 (in Russian); transl. in Russian Math. (Iz. VUZ) $51: 8,2007,74-77$.

[14] Elin, M., V. Goryainov, S. Reich, and D. Shoikhet: Fractional iteration and functional equations for functions analytic in the unit disk. - Comput. Methods Funct. Theory 2:2, 2002.

[15] Frolova, A., M. Levenshtein, D. Shoikhet, and A. Vasil'ev: Boundary distortion estimates for holomorphic maps. - Complex Anal. Oper. Theory 8:5, 2014, 1129-1149.

[16] Goryainov, V. V.: Fractional iterates of functions that are analytic in the unit disk with given fixed points. - Mat. Sb. 182:9, 1991, 1281-1299 (in Russian); transl. in Math. USSR-Sb. $74: 1,1993,29-46$.

[17] Goryainov, V.V.: Evolution families of conformal mappings with fixed points and the Löwner-Kufarev equation. - Mat. Sb. 206:1, 2015, 39-68 (in Russian); transl. in Sb. Math. 206:1-2, 2015, 33-60.

[18] Goryainov, V. V.: Holomorphic self-maps of the unit disc with two fixed points. - Presentation at XXV St. Petersburg Summer Meeting in Mathematical Analysis Tribute to Victor Havin (1933-2015), June 25-30, 2016, available at http://gauss40.pdmi.ras.ru/ma25/ index.php?page $=$ presentations.

[19] Goryainov, V. V.: Holomorphic mappings of the unit disc into itself with two fixed points. Mat. Sb. 208:3, 2017, 54-71 (in Russian); transl. in Sb. Math. 208:3, 2017, 360-376.

[20] Goryainov, V. V., and O. S. Kudryavtseva: One-parameter semigroups of analytic functions, fixed points and the Koenigs function. - Mat. Sb. 202:7, 2011, 43-74 (in Russian); transl. in Sb. Math. 202:7-8, 2011, 971-1000.

[21] Gumenyuk, P.: Parametric representation of univalent functions with boundary regular fixed points. - Constr. Approx. (to appear), https://doi.org/10.1007/s00365-017-9376-4.

[22] Ivanov, G., D. Prokhorov, and A. Vasil'ev: Non-slit and singular solutions to the Löwner equation. - Bull. Sci. Math. 136:3, 2012, 328-341.

[23] Koch, J., and S. Schleissinger: Value ranges of univalent self-mappings of the unit disc. J. Math. Anal. Appl. 433:2, 2016, 1772-1789.

[24] Koch, J., and S. Schleissinger: Three value ranges for symmetric self-mappings of the unit disc. - Proc. Amer. Math. Soc. 145:4, 2017, 1747-1761.

[25] Kufarev, P. P.: On integrals of simplest differential equation with moving pole singularity in the righ-thand side. - Tomsk. Gos. Univ. Uchyon. Zapiski 1, 1946, 35-48 (in Russian).

[26] Kufarev, P. P., V. V. Sobolev, and L. V. Sporyševa: A certain method of investigation of extremal problems for functions that are univalent in the half-plane. - Trudy Tomsk. Gos. Univ. Ser. Meh.-Mat. 200, 1968, 142-164 (in Russian). 
[27] Lind, J.: A sharp condition for the Loewner equation to generate slits. - Ann. Acad. Sci. Fenn. Math. 30, 2005, 143-158.

[28] Lind, J., and H. Tran: Regularity of Loewner curves. - Indiana Univ. Math. J. 65:5, 2016, 1675-1712.

[29] Löwner, K.: Untersuchungen über schlichte konforme Abbildungen des Einheitskreises. Math. Ann. 89, 1923, 103-121.

[30] Marshall, D. E., and S. Rohde: The Loewner differential equation and slit mappings. - J. Amer. Math. Soc. 18, 2005, 763-778.

[31] Poggi-Corradini, P.: Canonical conjugations at fixed points other than the Denjoy-Wolff point. - Ann. Acad. Sci. Fenn. Math. 25:2, 2000, 487-499.

[32] Pommerenke, Сh.: Boundary behaviour of conformal mappings. - Springer-Verlag, 1992.

[33] Pommerenke, Ch., and A. VAsil'ev: Angular derivatives of bounded univalent functions and extremal partitions of the unit disk. - Pacific J. Math. 206:2, 2002, 425-450.

[34] Pontryagin, L. S., V. G. Boltyanskit, R. V. Gamkrelidze, and E. F. Mishchenko: The mathematical theory of optimal processes. - Gosudarstv. Izdat. Fiz.-Mat. Lit., Moscow, 1961 (in Russian).

[35] Prokhorov, D., and K. SAmsonova: Value range of solutions to the chordal Loewner equation. - J. Math. Anal. Appl. 428:2, 2015.

[36] Prokhorov, D., and A. VAsiL'Ev: Singular and tangent slit solutions to the Löwner equation. - In: Analysis and Mathematical Physics, Trends in Mathematics, Birkhäuser Verlag, 2009, $455-463$.

[37] Roth, O., and S. Schleissinger: Rogosinski's lemma for univalent functions, hyperbolic Archimedean spirals and the Loewner equation. - Bull. Lond. Math. Soc. 46:5, 2014.

[38] VASIL'Ev, A.: On distortion under bounded univalent functions with the angular derivative fixed. - Complex Var. Theory Appl. 47:2, 2002, 131-147.

[39] Wong, C.: Smoothness of Loewner slits. - Trans. Amer. Math. Soc. 366:3, 2014, 1475-1496.

Received 6 June 2017 • Accepted 8 September 2017 